\title{
On Stress Measurement Errors in Biaxial Tensile Testing and the Impact on Yield Surface Identification
}

\author{
Sam Coppieters ${ }^{* \dagger}$, Tomoyuki Hakoyama ${ }^{\dagger}$, Daisaku Yanaga ${ }^{\dagger}$, Pascal Lava* $^{*}$ and \\ Toshihiko Kuwabara ${ }^{\dagger}$ \\ *Department of Mechanical Engineering, Catholic University College Ghent, Association KU Leuven, \\ Gebroeders De Smetstraat 1, B-9000 Gent, Belgium. \\ ${ }^{\dagger}$ Division of Advanced Mechanical Systems Engineering, Institute of Engineering, Tokyo University of \\ Agriculture and Technology, 2-24-16 Naka-cho, Koganei-shi, Tokyo 184-8588, Japan.
}

\begin{abstract}
In a biaxial tensile test on the sheet metal the calculation of the local stress tensor is based on the experimentally measured biaxial forces and surface strain at a well-defined position within the biaxial gauge area. The surface strains in this study are measured via our in-house digital image correlation system. A numerical method is proposed to estimate the influence of the experimental strain measurement error with respect to the stress error and the amount of plastic work per unit volume. In addition, the impact of the strain measurement error on the accuracy of yield surface identification is discussed.
\end{abstract}

Keywords: Sheet metal, biaxial testing, DIC

PACS: 07.05.Pj;62.20.-x;62.20.fq;07.10.Lw;07.10.Pz

\section{INTRODUCTION}

The introduction of slits in the arms of a cruciform specimen, as originally proposed by Kuwabara et al. [1] (Fig.1(a)), has been instrumental in avoiding geometrical constraints on the biaxial gauge area. Recently, Hanabusa et al. [2] proposed a method to further improve the accuracy of the testing method. Hanabusa et al. [2] numerically determined the optimal strain measurement position within the biaxial gauge area assuming that the strain can be measured without error. In this paper the purpose is to investigate the influence of the strain measurement error on the evolution of the stress error in biaxial tensile testing. It is clear that the accuracy and the precision of the strain measurement determines the accuracy of the stress points forming the contour of plastic work. Consequently, the accuracy of the identified yield function will also be affected. In this work, digital image correlation (DIC) is used to measure surface strains. First, the theoretical stress error as a function of the plastic work per unit volume is derived for different stress ratios. Then the focus is on the inclusion of the strain measurement error in the stress error assessment and the consequences for the identification of the yield function.

\section{EVOLUTION OF THE THEORETICAL STRESS ERROR}

The optimal strain measurement position guarantees a minimal mismatch between the local stress tensor and the global one. In [2] it was stated that this error is smaller than $2 \%$, however, there was no reference made to the amount of plastic work. In this section we investigate how the error is related to the amount of plastic work. To this purpose, the actual biaxial tensile test was simulated. Such a simulation can only be force controlled since the feedback loop (necessary to control the stress ratio) using the strains cannot be established in the finite element code. This means that the force ratio in the simulation is controlled rather than the stress ratio. Figure 1(a) shows the biaxial specimen used in this study and originally proposed by Kuwabara et al.[1]. The sheet metal used in this study is a dual phase steel with a tensile strength of $590 \mathrm{MPa}$ (JSC590Y) and an initial thickness of $1.2 \mathrm{~mm}$. In [2] it was concluded that the Yld2000-2d yield function [3] is an appropriate material model for this material under linear stress paths. Consequently, this material model is adopted in this study and the material parameters can be found in [2]. The arms of the cruciform are parallel to the RD and the TD of the sheet. The stress-strain curve from the uniaxial test in the RD is used as reference datum for work hardening. Furthermore, plane stress is assumed and the simulations are performed using the commercial 


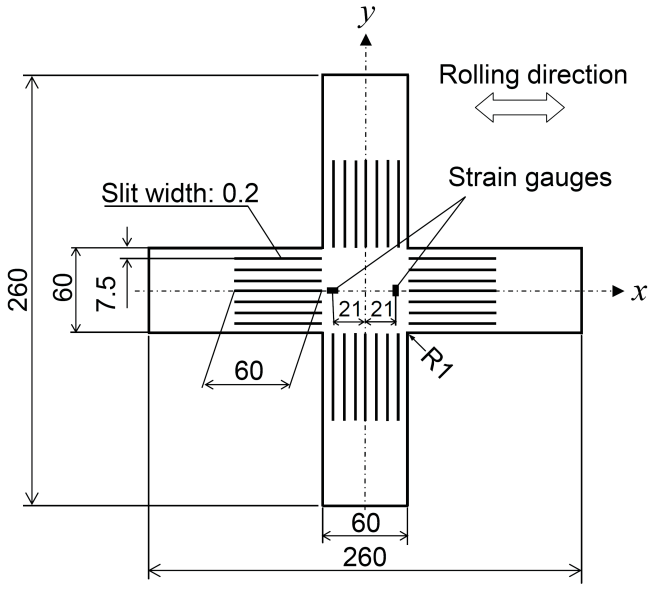

(a)

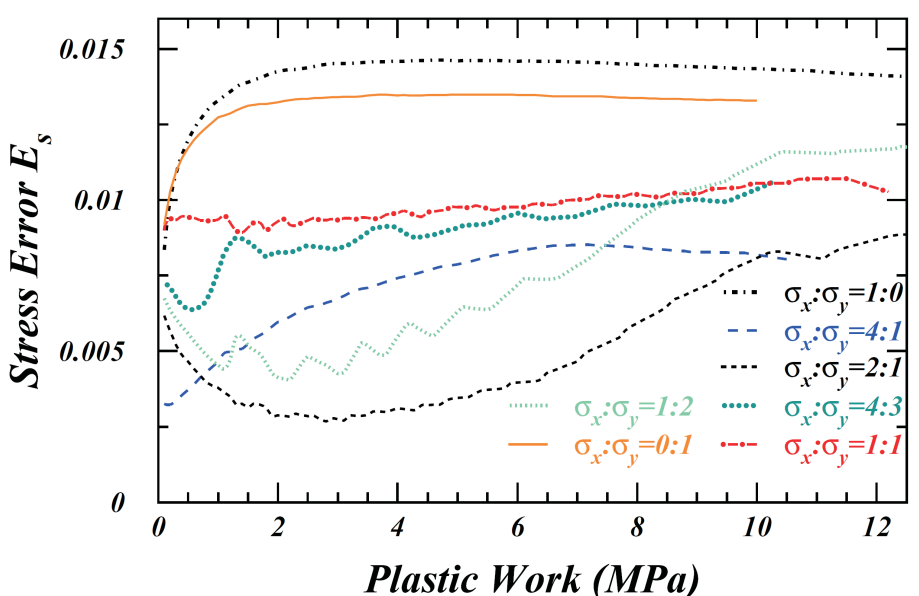

(b)

FIGURE 1. (a) Biaxial specimen after [1] (b) Theoretical stress error $E_{S}$ as a function of plastic work.

finite element code Abaqus/Standard. The stress error $E_{s}$ is defined as:

$$
E_{s}=\frac{\sqrt{\left(\sigma_{i j}^{G}-\sigma_{i j}^{L}\right)\left(\sigma_{i j}^{G}-\sigma_{i j}^{L}\right)}}{\sqrt{\sigma_{k l}^{L} \sigma_{k l}^{L}}}
$$

where $\sigma^{\mathbf{L}}$ is the local stress tensor and $\sigma^{\mathbf{G}}$ is the global stress tensor. $\sigma^{\mathbf{L}}$ is the local stress state calculated by the FE model at the strain measurement position. $\sigma^{\mathbf{G}}$ is constructed from the force and strain measurements which directly implies that the thickness stress and the shear stresses cannot be determined and therefore assumed to be zero $\left(\sigma_{12}^{G}=\sigma_{23}^{G}=\sigma_{31}^{G}=\sigma_{33}^{G}=0\right)$. The local stress state $\sigma^{\mathbf{L}}$ is assumed to be plane stress $\left(\sigma_{23}^{L}=\sigma_{31}^{L}=\sigma_{33}^{L}=0\right)$, and, consequently, the in plane shear stress $\left(\sigma_{12}^{L}\right)$ is taken into account. The strains $\left(\varepsilon_{x}, \varepsilon_{y}\right)$ are the total logarithmic strains at the positions prescribed by [2]. The plastic work $W_{p l}$ per unit volume calculated to track the evolution of the stress error reads as:

$$
W_{p l}^{G(L)}=\int_{0}^{\varepsilon_{i j}^{p l}} \sigma_{i j}^{G(L)} d \varepsilon_{i j}^{p l}
$$

where the superscript $\mathrm{G}$ or $\mathrm{L}$ denotes whether the global stress tensor or the local stress tensor is used, respectively. The elastic strains are removed from the measured total strain value assuming isotropic elastic behavior. A Young's modulus of 200GPa and a Poisson coefficient of 0.33 are used to construct the elasticity matrix. In order to be able to compare the stress errors at equal plastic work interpolation of the stress components is required. Figure 1(b) shows the theoretical stress error as a function of the plastic work $W_{p l}$ for the material under investigation. The maximum plastic work in this figure corresponds to a reference plastic strain $\varepsilon_{0}^{p l}=0.025$. It can be inferred that the largest stress error $E_{s}$ occurs for the stress ratios (1:0) and (0:1). This is not surprising since the specimen was not designed for this type of loading. Standard tensile specimens are of course superior in eliminating the stress error under uniaxial tensile testing. All other stress ratios exhibit a stress error smaller than or equal to $E_{S}=1 \%$. While not conclusive, this error evolution is expected to be dependent on the material response. Nevertheless, there is currently no reason to assume that the magnitude of this error would be larger for another material response as shown in [4]. Note that the errors in figure 1 are obtained by using the computed local strains. In the next section we investigate the same problem using strain values measured by DIC.

\section{INFLUENCE OF THE STRAIN MEASUREMENT}

In this section the influence of the strain measurement error on the stress error in biaxial tensile testing is investigated. To this purpose, the numerical procedure presented in [5] is adopted. The displacement fields from the sophisticated 

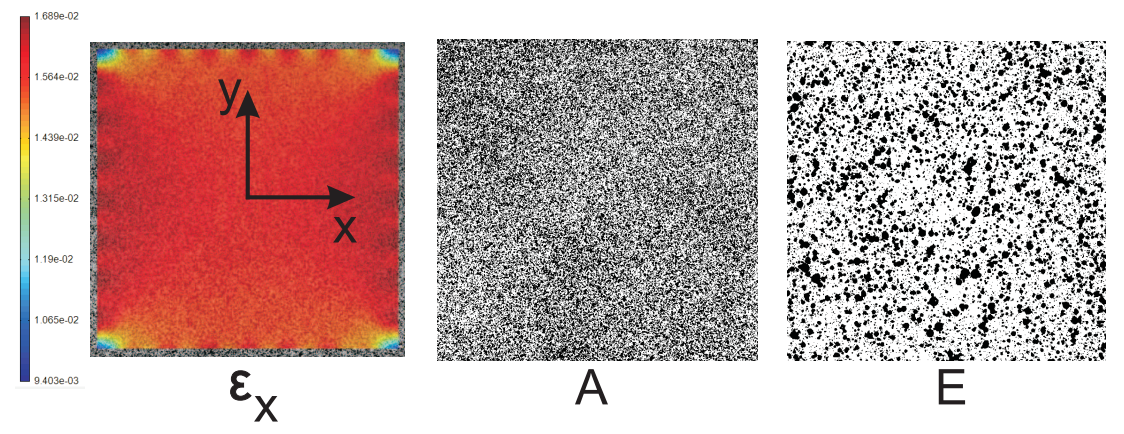

FIGURE 2. Left panel: strain component in the numerically deformed image (A) based on a simulation with $\left(F_{x}: F_{y}\right)=(1: 1)$ Middle panel: binary image pattern A. Right panel: binary image pattern E.

simulations in the previous section are used here to numerically deform images of speckled biaxial gauge areas. These deformed images are then post-processed in our DIC platform MatchID (http: / / www . matchid.org) to obtain the local strain tensor. The left panel of Figure 2 shows for example a strain component retrieved by 2D-DIC in a numerically deformed image. The deformation was based on the displacement field obtained by simulation of the biaxial tensile test with a force ratio of $\left(F_{x}: F_{y}\right)=(1: 1)$. Close to edges the strain concentrations due to slits, which act as stress raisers, are clearly visible. Finally, the stress errors can be calculated at equal plastic work as explained in the previous paragraph. In order to check the influence of the speckle pattern quality, two types of speckle patterns are used in this section. The middle panel of figure 2 shows a fine speckle pattern (A). The image shown in right panel of figure 2 exhibits a coarse speckle pattern (E). It can be inferred from figure 2 that pattern E exhibits featureless areas and an insufficient total coverage (28\%). In addition, 3 extra patterns (B,C and D) with similar global properties as pattern $\mathrm{A}$ (total coverage $\approx 45 \%$ and speckles with an average Feret diameter of $7 \mathrm{px}$ ) are manually created. The spatial imaging resolution of pattern $\mathrm{A}(\approx \mathrm{B}, \mathrm{C}$ and $\mathrm{D})$ and $\mathrm{E}$ shown in figure 2 are $0.086 \mathrm{~mm} / \mathrm{px}$ and $0.056 \mathrm{~mm} / \mathrm{px}$, respectively. The settings in MatchID 2D were the following: affine transformation, bicubic interpolation of the subset, bilinear interpolation of the strain window and the Zero-Normalized Sum of Squared Differences (ZNSSD) correlation algorithm. A subset and strain window of 30x30 pixels ${ }^{2}$ and $15 \times 15$ pixels $^{2}$ are used, respectively. The step size was chosen in such a way that the virtual strain gauge size matches the size of a typical strain gauge used in this experiment $\left(2 \times 2 \mathrm{~mm}^{2}\right)$. Figure 3 shows the results for the different stress ratios along with the theoretical stress errors found in the previous section. It can be inferred that if the strain measurement error is included, the stress error oscillates around the theoretical error. The errors introduced by similar patterns (A to D) are in very close agreement. The low-quality pattern E does not perform too bad, but inferior results such as for (1:2) at $W_{p l}=2 M P a$ could not be avoided. In general, it can be stated that the stress error $E_{s}$ can be kept smaller than $2 \%$ when the biaxial strains are measured with DIC provided that a sufficiently accurate strain sensor pattern is used. The final goal of biaxial tensile testing is usually to identify the shape and the evolution of the yield surface. In this section the numerical data generated in the previous section is used to identify the contours of plastic work. Since the parameters of the yield surface used in the FEA model are known, the quality of the identified yield loci at different levels of plastic strain can be assessed. This means that we can include the strain measurement error in the identification of the yield function. In addition, this identification is performed for eight different levels of plastic work with two different speckle patterns (A and E, see figure 2) to check the influence of the speckle pattern quality. Next, a stress state fitting procedure is used to find the best fit for the yield surface. The largest discrepancy was found for pattern $\mathrm{E}\left(\left(\sigma_{x}: \sigma_{y}\right)=(1: 2), W^{p l}=2 M P a\right)$. This can indeed be traced back to figure 3 which shows a large stress error $E_{s}$ for this particular amount of plastic work. In order to quantitatively compare the deviation between the shapes of the input work contours and the identified work contours, the following error metric can be used [6]:

$$
\delta_{r}=\sqrt{\frac{\sum_{i}^{N}\left(r^{i d}\left(\phi_{i}\right)-r^{i n}\left(\phi_{i}\right)\right)^{2}}{N}}
$$

where $\phi_{i}$ is the loading angle of the $i^{t h}$ stress point from the x-axis in the principal stress space, $r^{i n}\left(\phi_{i}\right)$ is the distance between the origin of the principal stress space and the $i^{t h}$ input stress point, $r^{i d}\left(\phi_{i}\right)$ is the distance between the origin of the principal stress space and the $i^{t h}$ identified stress point. It must be noted that $\delta_{r}$ is calculated here in the first 

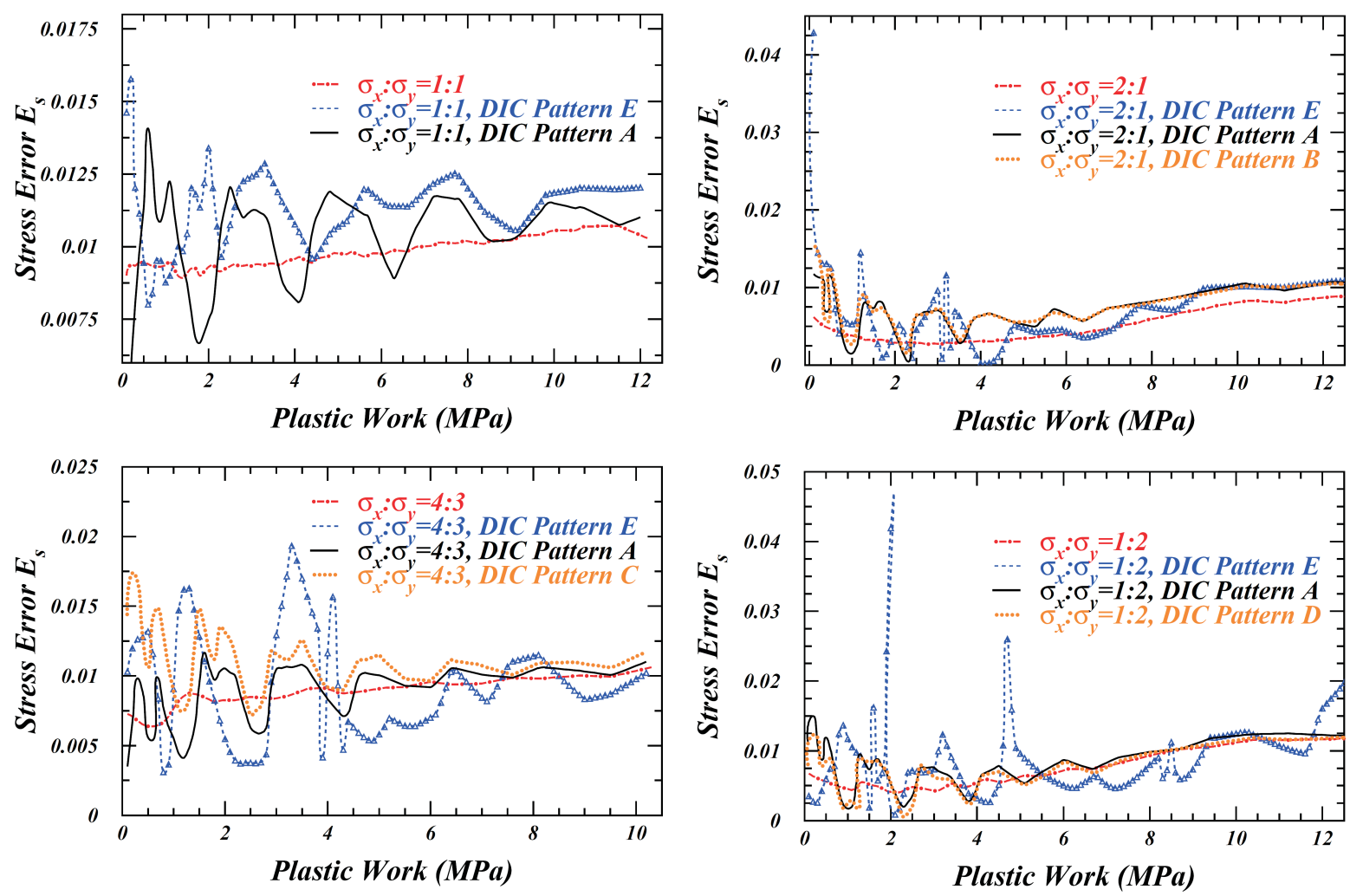

FIGURE 3. Stress error as a function of plastic work for different stress ratios.

quadrant of the stress space at equidistant loading angles with $\Delta \phi_{i}=5$ degrees. For all fine speckle patterns (A,B,C and D) $\delta_{r}$ equals approximately 0.005 . This value is found to be more or less independent of the plastic work. For the coarse speckle pattern a similar magnitude of $\delta_{r}$ was found, except for the earlier reported artifact at $W^{p l}=2 M P a$ where $\delta_{r}=0.02$.

In conclusion, the theoretical stress errors found in this study have an upper bound of approximately $1 \%$. It has been shown that if the surface strains are measured by DIC, the practical stress error oscillates around the theoretical stress error but never becomes larger than $2 \%$ provided that a sufficiently accurate speckle pattern is used. The effect of these stress errors on the identified yield surface in the first quadrant of stress space is studied and a value for the error metric proposed in [6] is provided.

\section{ACKNOWLEDGMENTS}

S.C. gratefully acknowledges the support of Japanese Society for the Promotion of Science (JSPS) through the postdoctoral JSPS fellowship.

\section{REFERENCES}

1. T. Kuwabara, S. Ikeda, T. Kuroda, J. Mater. Process. Technol. 80 - 81, 517-523 (1998)

2. Y. Hanabusa, H. Takizawa, T. Kuwabara, J. Mater. Process. Technol. 213, 961-970 (2013)

3. F. Barlat, Brem, J.C., Yoon, J.W., Chung, K., Dick, R.E., Lege, D.J., Pourboghrat, F., Choi, S.H., Chu, E., Int. J. Plasticity 19, 1297-1319 (2003)

4. Y. Hanabusa, H. Takizawa, T. Kuwabara, Steel Res. Int. 81, 1376-1379 (2010)

5. Y. Wang, P. Lava, S. Coppieters, M. De Strycker, P. Van Houtte and D. Debruyne Strain 48, 453-462 (2012)

6. T. Kuwabara and F. Sugawara., Int. J. Plasticity 45, 103-118 (2013) 